\title{
FIELD QUALITY AND STABILITY OF PERMANENT MAGNET QUADRUPOLES
}

S. Herb

Laboratory of Nuclear Studies

Cornell University

Ithaca, NY \$4853

\section{Aostract}

We plan to increase the peak luminosity of the Cornell Electron Storage Ring by providing tighter vertical focussing at the interaction points. The final focussing devices will be large permanent magnet quadrupoles operating inside the 10-15 ko solenoidal field of the experimental detector. We present the results of tests demonstrating that quadrupoles with the required field quality and resistance to cemagnetization can be built from commercially available Rare Eartin Cobalt magnets.

\section{Introduction}

As part of a luminosity upgrade program at the Cornell Electron Storage Ring (CESR) we are building large permanent magnet quadrupoles with $10 \mathrm{~cm}$ bore, $120 \mathrm{~cm}$ length, and $7.5 \mathrm{kG}$ pole-tip field. They will operate inside the 10-15 kG field of the CLEO detector solenoid, permitting tighter vertical focussing of the beam without an increase in vertical chromaticity. It is possible to use aligned Rare Earth Cobalt (REC) material for this application because of its extreme resistance to demagnetization by fields perpendicular to the preferred direction of magnetization, or "easy axis". In addition, because of the limited energy range over which CESF is operated an adjacent electrically powered quadrupole may be used to compensate the decrease in focussing strength of the REC quadrupole as the beam energy is raised.[1]

Requirements on field quality and stability in interaction region quadrupoles are especially severe. our design goal has been for multipole fields to be no greater than several parts in $10^{4}$ of the quadrupole field at $4 \mathrm{~cm}$ ( $80 \%$ of pole-tip radius), which is the maximum radius not shadowed by other apertures in the machine. The problem has been to establish whether this field quality can be achieved with commercially available REC magnets and whether it will be stable during extended exposure to the $10-15 \mathrm{kG}$ solenoidal field. This report describes measurements on $\mathrm{CO}_{5} \mathrm{Sm}$ magnets from several manufacturers and on a prototype 16 segment quadrupole.

The quadrupoles are built according to Halbach's prescription for segmented REC quads (Fig. 1), resulting in a pole-tip field, $B=2 B_{r} *(1-r 1 / r Z) * K_{2}$ (in Halbach's notation), with ratio of inner to outer radius $r 1 / r 2=0.5$ and $K_{2}=0.85 \quad\left(K_{2}=1.00\right.$ for

infinitesimal segmentation with no air gaps between the inner and outer radius).[2] For ideal magnetic material the next lowest multipole allowed by symmetry for the 16 segment quadrupole is $n=18$ (where $n=2$ is quadrupole); therefore all important defects result from deviations of the REC material from ideal behavior.

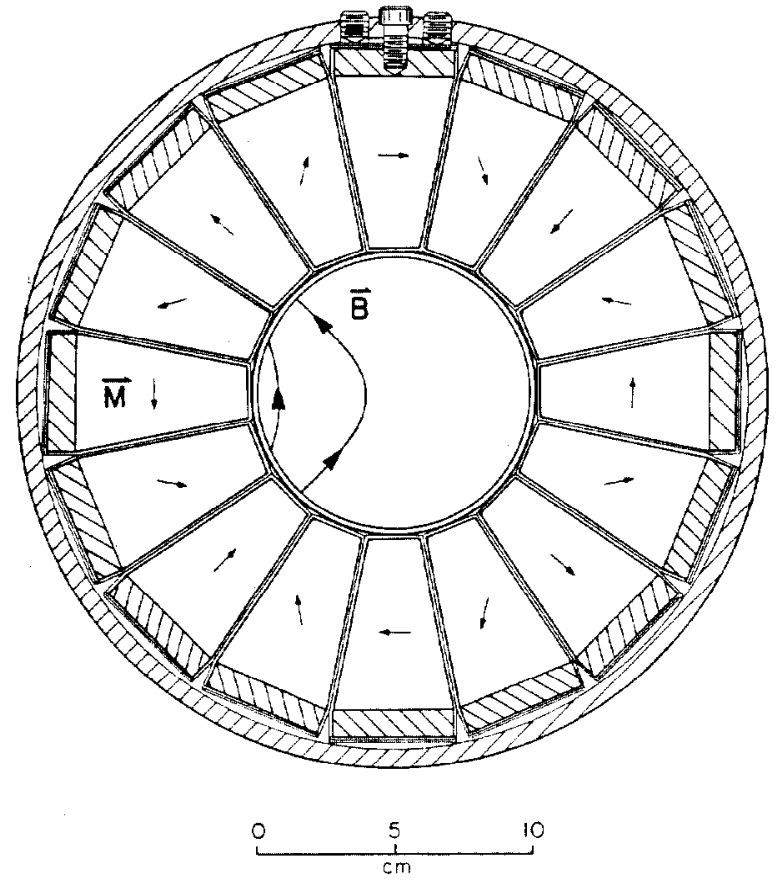

0920485-001

Fig. 1 Direction of magnetization $M$ and magnetic field lines $B$ in a 16 segment REC quadrupole.

We have performed tests on magnet samples of HICOREX 96B (from Hitachi Magnetics Corp.) and VACOMAX 170 (from Vacuumschmelze GMBH). These (mostly) $\mathrm{Co}_{5} \mathrm{Sm}$

magnets may be characterized to first approximation by a few numbers (Fig. 2):

$\begin{array}{ll}\text { Magnetization } & \mu_{0} M \simeq B_{r} \simeq 9 \mathrm{kG} \\ \text { Permeability } & \mu_{\|} \simeq \mu_{\perp} \simeq 1.03-1.06 \\ \text { Coercivity } & \mu_{0} H_{c 1} \simeq-15,-20 \mathrm{kG}\end{array}$

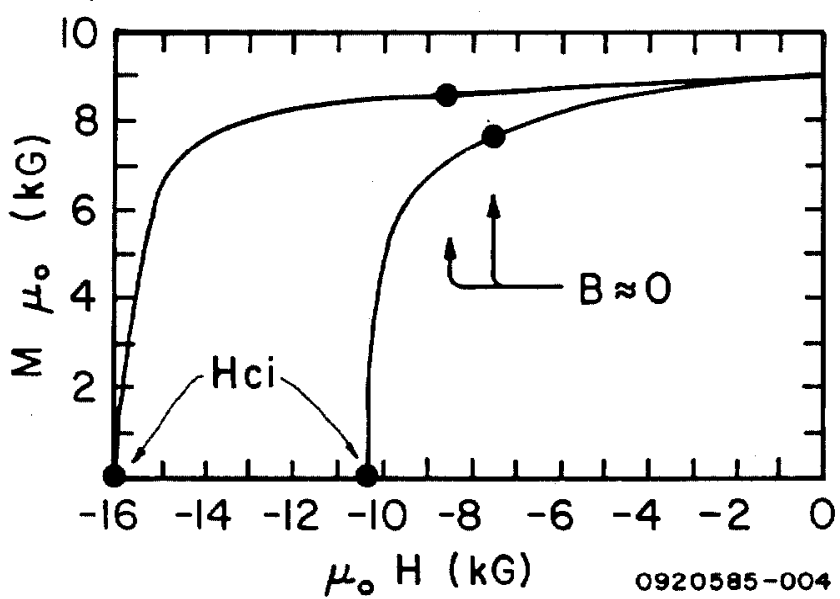

Fig. 2 Schematic curves for magnetization vs. H field in the easy axis direction for $\mathrm{CO}_{5} \mathrm{Sm}$ magnets with good and substandard coercivity. $H_{c i}$ is the field at which the magnetization has dropped to zero. 
Once one is interested in effects at the percent level, however, all of the famlliar complications of magnetic material, such as hysteresis, reappear. We will present our results as a sort of user's guide to these complications.

\section{Piece to Piece Variation}

Variations in magnituce of magnetization of $\pm 2 \%$ and $\pm 2^{\circ}$ in direction are achievabie with standard but careful industrial practices and some selection of the finished pieces. This introduces multipole errors at a level of typically $2 \% / \sqrt{N}$ or about $1 / 2 \%$ for the 16 segment quadrupoles.

\section{Self-Induced Multipoles}

The field of the magnets acting on their own nonunity permeability induces higher multipole moments. This is easily seen in Fig. 1; the field of the quadrupole enhances the magnetization of segments with radial easy axis orientation and depresses it for those with tangential orientation. This turns out to result in a codecapole moment at the $1 / 2 \%$ level.

Both effects I and II are easily compensated by the magnetic tuning procedure suggested by Halbach in which small motions of the magnet blocks are used to null the measured multipole moments.[3] For the 16 segment quadrupole, using only radial motion of the segments, it is easy to construct a set of orthogonal vectors which give independent control of all multipoles from $n=3$ (sextupole) through $n=10$ even. Figure 3 shows the multipole content for the prototype ring before and after tuning, as measured using a rotating coil apparatus. Typical radial motions were $1 / 2 \mathrm{~mm}$ or about 1\% of the pole-tip radius. Using this scheme the dipole and sextupole moments cannot be independently adjusted; the dipole may be considerably reduced by matching of pieces before assembly or by arranging that the dipole moments of adjacent slices of the quadrupole cance:.

\section{Irreversible Demagnetization}

Anti-Parallel Fields: When the magnets are assembled into the quadrupole the local field observed by some of the magnetic material changes drastically. For quacrupoles with ratio of inner to outer radius of 0.5 the $B$ field is reduced almost to zero $(H=-M)$ in

some regions; this can be seen from Halbach's quadrupole formula and inspection of the field direction near the pole-tips. For inner radius much less than outer some, material will be pushed far into the third quadrant of the BH plane. Under these conditions some demagnetization will occur. Figure $4 a$ shows the demagnetization for test pieces exposed to $\mu_{0} \mathrm{H}=-10 \mathrm{kG}$, as measured before and after with a Helmholtz coil. The typical loss of magnetization is 3-6\% and one piece falls by 50\%. This demagnetization can obviously be compensated by tuning but stability problems, discussed below, may remain. The problem can be minimized by requiring high coercivity, however, this is not trivial since coercivity is the most difficult property to control during production of the magnets.
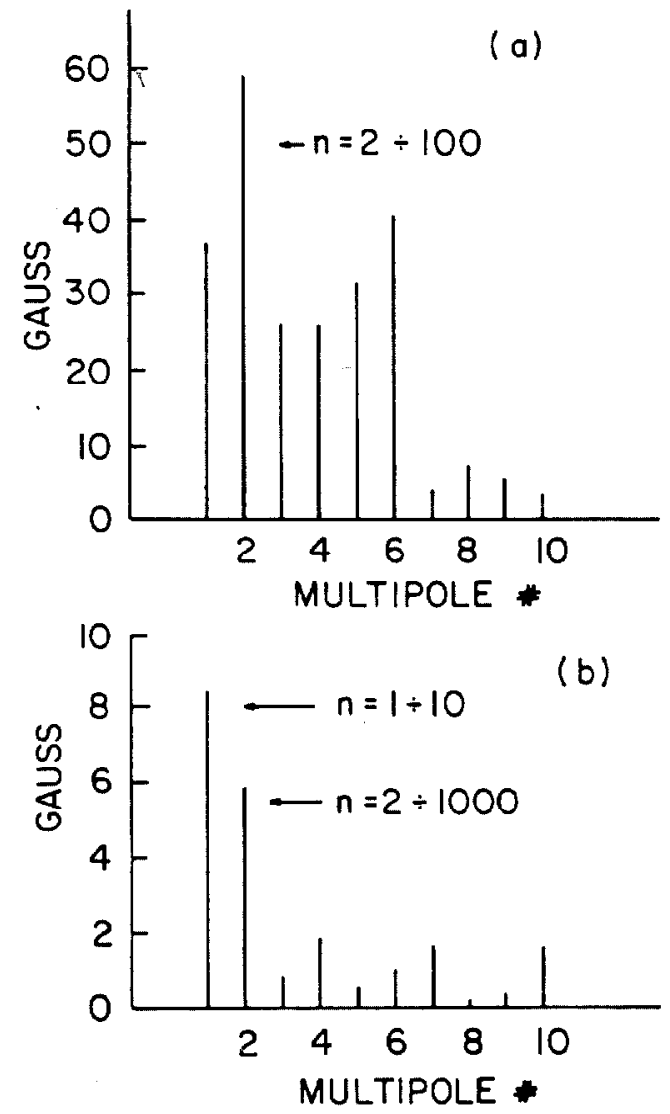

Fig. 3 a) Multipole content of prototype quadrupoie measured with rotating coil apparatus before tuning. Multipole fields are evaluated at $4 \mathrm{~cm}$ radius. b) the same, after tuning. All moments except the dipole are less than 3 parts in 10,000 of the quadrupole.

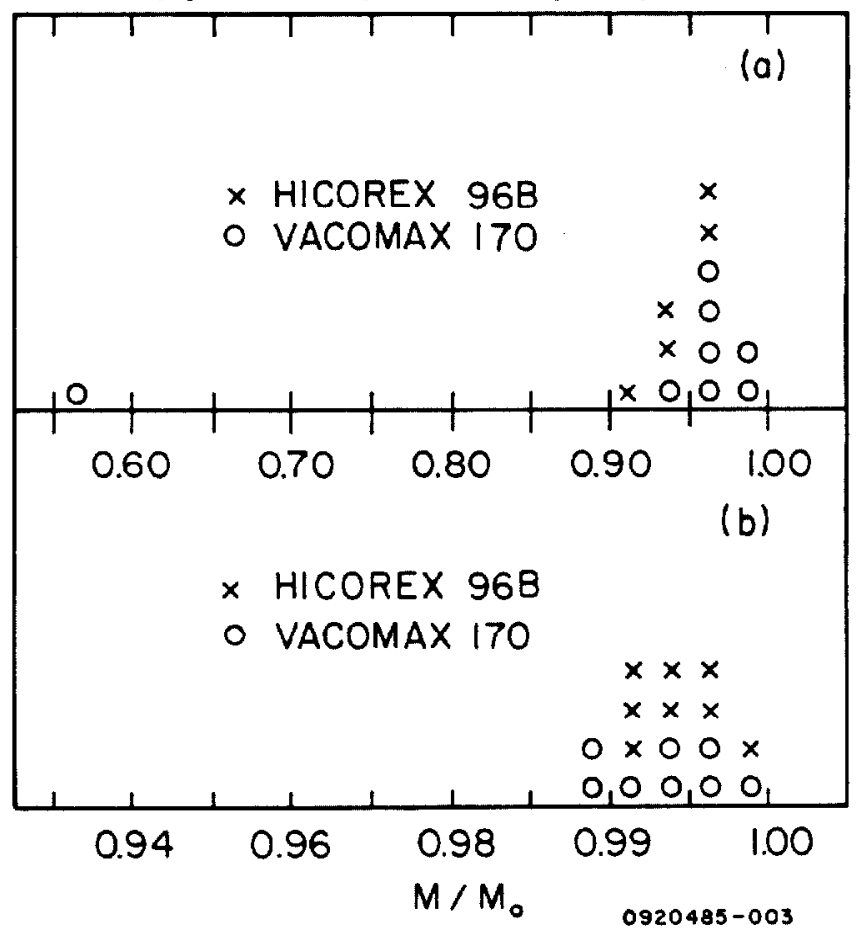

Fig. 4 a) Histogram for REC magnet samples of the ratio of final to initial magnetization for exposure to a field $\mathrm{H}=-10 \mathrm{kG}$ in the easy axis direction, as measured using a Helmholtz coll apparatus b) The same, but before and after exposure to a transverse field of $15 \mathrm{kG}$. 


\section{Irreversible Demagnetization}

Perpendicular Fields: Despite the resistance of the REC material to transverse magnetic fields, small decreases in magnetization also occur on exposure to fields of 10-15 kG. Eigure $4 \mathrm{~b}$ shows the demagnetization of pieces exposed to $15 \mathrm{kG}$ again measured before and after with a Helmholtz coil. The decrease is $0-1 \%$. This may be qualitatively understood by realizing that the alignment of grains in the REC

material is far from ideal, with many grains 15 or $20^{\circ}$ away from the average easy axis. These grains will contribute to the magnetization along the easy axis but will also see a substantial component of a transverse field, so that low coercivity grains may be reversed.

A series of tests was performed in which the prototype quadrupole was exposed to transverse fields of 10-15 kG, with multipole content measured before and after each exposure using the rotating coil. At $15 \mathrm{kG}$ the quadrupole field decreased by $0.7 \%$ and multipoles appeared at a level consistent with substantial segment to segment variations in the decreasc. After this initial change no additional change was observed during a 2 week exposure to $12 \mathrm{kG}$, so exposure to high field and retuning to compensate changes should be an adeçuate solution.

\section{v. Stability}

Permanent magnets are not permanent (in an open magnetic circuit); the magnetization decays roughly as the logarithm of time, rapidly at first, then more and morc slowly. A rough picture is that some distribution o: energy barriers to grain reversal is being joggled by thermal fluctuations. The standard method for "stabilizing" this aging process is to heat the magnet after magnetization; this is effectively a speeded up aging which decreases the magnetization but also slows the rate of change. The residual change may be less than 1\%/year but depends crucially on the operating Foint of the magnet on the MH plane, or more particularly on the slope of $M(H)$ near the operating point.[4] A magnet sitting on or near the precipitous fall in magretization at the coercive limit will have poor stability against aging, temperature change, and changes in the external magnetic field, so it is desirable that all magnetic material operate well away from this region.

Our solution to this requirement is to measure coercivity for all incoming pieces by subjecting them to a field rore negative than they will see in operation and measuring the resulting demagnetization. our specification, tight by industry standards, is a maximum $6 \%$ demagnetization for exposure to $\mu_{0} \mathrm{H}=-10 \mathrm{kO}$ $(B=-1 k G)$. We then remagnetize the pieces in a $15 k G$ field (the highest conveniently available) which restores them to their initial magnetization.

It is also important that the heat stabilization be performed when the magnets are in a field similar to that which they will see during operation; we will

expose our quadrupoles to about $120^{\circ} \mathrm{C}$ after assembly of 16 segment rings.

We do not have a complete set of measurements on the effects of aging on the time scale of a year nowever, our prototype quadruoole was recently remeasured after being neglected for the better part of a year and changes in multipole moments were close to the level of measurement error, 1-2 parts in $10^{4}$ of the quadrupole field.

\section{Radiation Damage}

Several measurements of radiation damage due to high energy hadrons have been reported $[5,6]$. At doses of several Gigarads decreases in magnetization in the range 1-20\% were observed. We have exposed several small magnets to a dose of 1 Megarac in a Cobalt 60 chamber with no change in magnetizaticn observed at the $1 / 2 \%$ level.

\section{Conciusion}

High strength quadrupoles with storage ring quality can be built from commercially available $\mathrm{CO}_{5} \mathrm{Sm}$

magnets. During assembly of such quadrupoles (and exposure to high transverse fields) changes in local magnetization will occur at the several percent level so an iterative procedure of assembly, exposure, and tuning of multipole content is desirable for achieving best field quality and stability. Special attention should be given to the coercive properties of the REC material over the range of operating points it will experience in service.

\section{Acknowledgements}

I wish to acknowledge useful discussions with K. Halbach, J. Seeman, J. Spencer, H. Marik of Vacuumschmelze GMBH, and many other people at Hitachi Magnetics and Vacuumschmelze. John Walters and Gerry Jackson contributcd to the assembly and measurement work. This work was supported by the Laboratory of Nuclear Studies at Corneli University through a grant from the National Science Foundation.

\section{References}

1. S. Peck and J. Seeman, CBN 82-36 (Cornell LNS Technical Note)

2. K. Halbach, Nucl. Instr. and Meth. 169, 1, 1980

3. K. Halbach, Nucl. Instr. and Meth. 198, 213, 1982

4. E. Adler and H. Marik, Paper No. VII-3 at 5 th International Workshop on Rare-Earth-Cobalt Permanent Magnets and their Applications; Rnanoke VA, June 1981 (Book by Univ, of Dayton, KL-365, Dayton, Ohio 45469)

5. F. Coninckx et al., 'CERN/SPS 83-1 (EBS)

6. E. Blackmore, TRIUMFH (paper submitted to this conference) 